\title{
Temporal bone computed tomography findings in bilateral sensorineural hearing loss
}

\author{
D E Bamiou, P Phelps, T Sirimanna
}

\begin{abstract}
Aim-To examine the yield of computed tomography (CT) of the temporal bones when investigating sensorineural hearing loss (SNHL) and to identify factors associated with CT findings.

Methods-Retrospective analysis of 116 consecutively investigated children with bilateral SNHL at the audiology department of Great Ormond Street Hospital, London. Main outcome measures were CT results, hearing loss parameters, history, and clinical examination.

Results-A total of $33(28.4 \%)$ CT scans were identified as abnormal. Children with profound and/or progressive hearing loss and/or craniofacial abnormalities were more likely to have an abnormal CT scan and together accounted for 25 abnormal CT scans. Sex, consanguineous parents, or family history of SNHL were not associated with CT findings. Dilated vestibular aqueduct was significantly correlated with the presence of progressive SNHL.

Conclusions-All children with SNHL should undergo radiological investigation of the petrous bones/inner ear; abnormalities are more likely to be found in cases with craniofacial abnormalities, or profound or progressive hearing loss. The decision whether to perform a CT or magnetic resonance imaging will depend on scanner availability, expertise, and management considerations, but cochlear implant candidates will require both. (Arch Dis Child 2000;82:257-260)
\end{abstract}

Keywords: computed tomography; sensorineural hearing loss

Computed tomography (CT) of the petrous bones is a first line recommended investigation of sensorineural hearing loss (SNHL). ${ }^{1}$ In cases of bilateral SNHL, the CT scan may be abnormal in $6.8 \%{ }^{2}$ to $12.8 \%,{ }^{3}$ and in up to $30 \%$ of cochlear implant candidates. ${ }^{4}$ However, some authors ${ }^{3}$ argue that CT may be unjustified for children with stable hearing impairment and no other abnormalities.

Our aim was to examine the CT yield in the investigation of SNHL in children, to identify factors correlated with CT findings, and to assess the effect of the CT findings on management.

Materials and methods

The study included patients with SNHL who had been investigated regarding the aetiology of their hearing loss at the audiology department, Great Ormond Street Hospital between January 1996 and June 1998.

Criteria for inclusion in the study were:

- Bilateral SNHL, defined as:

- air conduction thresholds in the standard pure tone audiogram (PTA), averaged across $500,1000,2000$, and $4000 \mathrm{~Hz}$, worse than $20 \mathrm{~dB}$ hearing loss in the better hearing ear or

- high frequency hearing loss, with low and mid frequency thresholds better than $20 \mathrm{~dB}$ hearing loss and average threshold at 4000 and $8000 \mathrm{~Hz}$ worse than $20 \mathrm{~dB}$ hearing loss in the better hearing ear

- air-bone conduction gap of $10 \mathrm{~dB}$ hearing loss or less, as identified by masked bone conduction audiometry, in both ears.

- Bilateral SNHL, as above, and fluctuating conductive type hearing loss consistent with the presence of otitis media with effusion.

Hearing loss was classified by averaging air conduction thresholds across 500, 1000, 2000, and $4000 \mathrm{~Hz}^{1}$ of the better hearing ear, as: mild, $>20$ and $\leqslant 40 \mathrm{~dB}$ hearing loss; moderate, $>40$ and $\leqslant 70 \mathrm{~dB}$ hearing loss; severe, $>70$ and $<95 \mathrm{~dB}$ hearing loss; profound, $\geqslant 95$ $\mathrm{dB}$ hearing loss.

High frequency SNHL was defined as average threshold across $500-4000 \mathrm{~Hz}$ better than $20 \mathrm{~dB}$ hearing loss, and average threshold of 4000 and $8000 \mathrm{~Hz}$ over $20 \mathrm{~dB}$ hearing loss.

CT scans of the petrous bones and internal auditory meatuses were performed on a Siemens Somaton +4 scanner in $1 \mathrm{~mm}$ serial axial and coronal cuts. Axial scans were oriented parallel to the infraorbital-meatal line, while coronal scans were tilted at a $105^{\circ}$ angle to this line.

\section{STATISTICAL ANALYSIS}

The Statistical Package for the Social Sciences (SPSS) was used to analyse the study results. The results were summarised as means, medians, and percentages. Subgroups within the study population were compared by $\chi^{2}$ and Mann-Whitney tests. A p value of 0.05 or less was accepted as indicating statistically significant results.

\section{Results}

Review of the records identified 116 children with bilateral SNHL who underwent CT investigation during the period of the study. Nine children had progressive hearing loss $(7.8 \%)$. Males predominated in the sample (70 males $(59.8 \%)$ and 46 females $(40.2 \%)$ ), but there was no significant difference in the degree of the SNHL between boys and girls (Mann- 
Table 1 Epidemiological data summary

\begin{tabular}{ll}
\hline Total number & 116 \\
Age (years), mean (SD) & $9.1(4.47)$ \\
Age at diagnosis (years), mean (SD) & $3.2(2.5)$ \\
Male, n (\%) & $70(59.8)$ \\
Progressive, n (\%) & $9(7.8)$ \\
Parents related, n (\%) & $19(16.4)$ \\
Family history of SNHL, n (\%) & $34(29.3)$ \\
\hline
\end{tabular}

Whitney test, $\mathrm{p}>>0.05)$. The child's parents were related in 19 cases $(16.4 \%)$. There was a first or second degree relative with documented SNHL of possible genetic origin in 34 cases $(29.3 \%)$. Table 1 summarises these results.

There were 38 cases $(32.8 \%)$ with profound SNHL (table 2). The severity of SNHL (as an average or as percentage of profound cases, for example) was not significantly different between the two sexes ( $p>>0.05)$.

All 116 children had CT scans of the petrous pyramids and internal acoustic meatuses (IAM). A total of $33 \mathrm{CT}$ scans (28.4\%) were reported as showing abnormalities of the inner ear, internal auditory meatuses, or other associated structures. Table 3 summarises these results.

The diagnosis of the aetiology of hearing loss was based on CT findings and other investigations as well as on the family history of hearing loss, the child's medical history, and audiometric patterns. Identification of factors that might have caused the hearing loss was possible in 93 cases $(80.2 \%)$ and unknown in $23(19.8 \%)$. Of the 93 cases with an available (presumptive) diagnosis:

- $51(44 \%)$ were classified as genetic: 20 indicating autosomal recessive inheritance, nine indicating autosomal dominant, four indicating mitochondrial, one indicating $\mathrm{X}$ linked, five chromosomal, and 12 genetic syndromic (three

Table 2 SNHL characteristics

\begin{tabular}{ll}
\hline & $n(\%)$ \\
\hline Mild & $14(12.1)$ \\
Moderate & $24(20.7)$ \\
Severe & $32(27.6)$ \\
Profound & $38(32.8)$ \\
High frequency SNHL & $3(2.6)$ \\
Missing data $^{\star}$ & $5(4.3)$
\end{tabular}

*Missing data applies to cases in which the degree of SNHL was not fully established (information only from auditory brainstem response (ABR) - all five cases with $\mathrm{ABR}$ thresholds exceeding $70 \mathrm{~dB}$ nHL).

Table 3 CT scan results

\begin{tabular}{lll}
\hline CT report & $n$ & $\%$ \\
\hline Normal & 83 & 71.6 \\
Common cavity deformity & 1 & 0.9 \\
Mondini & 3 & 2.6 \\
Mondini and dilated vestibular aqueduct & 4 & 3.4 \\
Dilated vestibular aqueduct & 6 & 5.1 \\
Narrow IAM & 3 & 2.6 \\
Abnormal vestibule/semicircular canals & 7 & 6.0 \\
Abnormal cochlea, vestibule, and IAM & 5 & 4.3 \\
Cerebral abnormalities & 1 & 0.9 \\
Miscellaneous $^{\star}$ & 3 & 2.6
\end{tabular}

The common cavity deformity consists of absent or dilated cochlear basal coil widely communicating with the vestibule. "Mondini" describes a cochlea with a basal turn and a distal sac. Dilated vestibular aqueduct refers to middle third diameter of more than $1.5 \mathrm{~mm}$.

*Two cases with skull base deformity and one with high jugular bulb on one side. branchio-oto-renal syndrome (BOR) - all with Mondini type cochlea; eight Pendred syndrome-four with dilated vestibular aqueduct and four with dilated vestibular aqueduct and Mondini type cochlea; and one Usher I syndrome ${ }^{\star}$ )

- $10(8.6 \%)$ congenital syndromic (four CHARGE, one VATER RAPADILINO*, one case first arch syndrome, four unclassified)

- five $(4.3 \%)$ congenital-unclassified (sporadic or autosomal recessive, all with abnormal CT scans)

- $\operatorname{six}(5.2 \%)$ following prenatal complications

- seven (6\%) following perinatal complications

- two $(1.7 \%)$ ototoxic (gentamycin)

- $\operatorname{six}(5.2 \%)$ post-meningitic

- four $(3.4 \%)$ post-viral infections

- two $(1.7 \%)$ post-traumatic.

*Note: Usher I syndrome is an autosomal recessive disorder characterised by early onset retinitis pigmentosa, severe to profound hearing loss, and absent vestibular responses. ${ }^{7}$ The acronym CHARGE describes the association of colobomata, heart defect, atresia of the choanae, retarded growth/development, genital hypoplasia, and ear anomalies or deafness. The acronym VATER describes the association of vertebral defects, anal atresia/stenosis, tracheooesophageal fistula, radial defects, and renal anomalies. ${ }^{5}$ The acronym RAPADILINO describes radial defects, absent/hypoplastic patellae and high/cleft palate, diarrhoea (and dislocated joints), little size, a long/slender nose, and normal intelligence, with hearing loss also a feature. ${ }^{6}$

Table 4 summarises these results (diagnostic labels against number of positive CT findings in each subgroup).

The presence of progressive hearing loss was a significant predictor of positive (abnormal) CT outcome ( $p=0.01564$, Fisher's exact test). Six of nine cases $(66.6 \%)$ with progressive SNHL had a positive CT scan, while only 26 of $103(25.24 \%)$ children with nonprogressive hearing loss had a positive CT scan. (Note: there were four missing data regarding progressiveness of SNHL.) Cases with dilated vestibular aqueduct (DVA), with or without other abnormalities, were more likely to have progressive SNHL than the rest of the sample population ( $\mathrm{p}=0.02397$, Fisher's exact test). Three of nine cases $(33.3 \%)$ with DVA had a progressive hearing loss but

Table 4 Aetiological diagnosis of bilateral SNHL and abnormal CT scans

\begin{tabular}{lll}
\hline Diagnostic labels & $n(\%)$ & $\begin{array}{l}\text { Abnormal CT } \\
\text { scan }(n)\end{array}$ \\
\hline Genetic & $51(44)$ & 21 \\
Congenital syndromic & $10(8.3)$ & 6 \\
Congenital non-syndromic & $5(4.3)$ & 5 \\
Prenatal & $6(5.2)$ & 0 \\
Perinatal & $7(6)$ & 0 \\
Ototoxic & $2(1.7)$ & 0 \\
Post-meningitic & $6(5.2)$ & 1 \\
Post-childhood infections & $4(3.4)$ & 0 \\
Post-traumatic & $2(1.7)$ & 0 \\
Unknown aetiology & $23(19.8)$ & 0 \\
Total & 120 & $33(27.5 \%)$ \\
\hline
\end{tabular}


only six of 107 cases (5.6\%) without DVA had progressive hearing loss.

Children with profound SNHL were more likely to have an abnormal CT scan ( $p=0.00583)$, as 17 of $38(44.7 \%)$ profoundly hearing impaired children had an abnormal CT scan, while of the remaining 75 children with mild, moderate, severe, and high frequency SNHL, only $15(20 \%)$ had abnormal scans. Craniofacial abnormality was also significantly correlated with an abnormal CT scan $(p=0.00794):$ nine of $16(56.25 \%)$ children with craniofacial abnormalities had a positive CT scan, but only 24 of 100 (24\%) children with no craniofacial abnormality had an abnormal CT scan. Neither consanguineous parents, nor a family history of hearing loss, nor sex of the patient were significantly correlated with an abnormal CT scan ( $p>0.05)$.

\section{Discussion}

We found a $28.4 \%$ yield of CT in identifying abnormalities relevant to the cause of SNHL in children in our tertiary tier audiology department. This is similar to the findings of Woolford et al, ${ }^{4}$ who reported $29.5 \%$ abnormal CT scans in adults and children awaiting cochlear implantation. Shusterman et al, ${ }^{3}$ however, reported a yield of $12.85 \%$, while an earlier study by Zalzal et al reported an incidence of $6.8 \%$ abnormal CT findings in children with SNHL. This wide range of CT yields may be explained partly by the improvement of imaging techniques and better understanding of SNHL related inner ear abnormalities over the years, as well as by the different populations targeted by the different studies.

The male preponderance in children with SNHL (59.8\% in our study) has been commented on by several authors. ${ }^{8}{ }^{9}$ We found no statistically significant sex difference, in terms of either severity of hearing loss or CT findings. A convincing explanation for the male preponderance is still lacking, although possible explanations include preferential referrals of boys to tertiary audiology centres, male children being more susceptible to hearing damage, and genetic differences.

DVA was the most common CT abnormality in our series (10 of 116 cases), as in other studies. ${ }^{10}$ DVA was an isolated finding in six $(60 \%)$ and an associated finding in four $(40 \%)$ of our cases, in contrast with the findings of Valvassori and Clemis, ${ }^{11}$ that DVA in association with other cochlear abnormalities is more prevalent than as an isolated finding. Three of our nine cases with DVA had progressive hearing loss (information was missing regarding progressiveness of hearing loss in one case), similar to the report of Antonelli et al. ${ }^{12}$ Cases with DVA were more likely to have progressive hearing loss than the rest of the sample population, and this association was statistically significant. This information was particularly relevant in terms of management, as hearing thresholds may deteriorate after trauma when DVA is present, ${ }^{12}{ }^{13}$ so we advised all patients with DVA to avoid contact sports.

Mondini-type dysplasia, defined as presence of a cochlea with a normal basal turn and a distal sac, ${ }^{14}$ was the third commonest abnormality, as an isolated finding in three cases, and in association with dilated vestibular aqueduct in four cases. Jackler et $a l^{15}$ found this abnormality, described as incomplete partition, in 41 of 98 cases with abnormal CT scans $(41 \%)$, and proposed that this deformity corresponds to an arrest in development during the seventh week in utero. These authors also reported the presence of dilated vestibular aqueduct in 10 of 41 ears (24\%), but estimated that DVA was under reported in their study, as lateral polytomograms were not available for all their patients.

Four of our children with DVA and four children with DVA and Mondini were thought to have Pendred's syndrome, an autosomal recessive condition that consists of congenital hearing loss and thyroid goitre or positive perchlorate test. ${ }^{16}$ Phelps et $a l^{16}$ reported dilated vestibular aqueduct present in all 120 cases with Pendred's syndrome they reviewed, while Mondini-type dysplasia was also a common finding. Branchio-oto-renal syndrome (BOR) is an autosomal dominant disorder, with hearing loss, preauricular pits, renal anomalies, branchial fistulae, pinna, and external auditory canal deformities as major features. ${ }^{17}$ Our three cases with BOR syndrome had a Mondini-type abnormality of the cochlea, in accordance with previously published reports. ${ }^{17}$

Abnormalities of the semicircular canals were the second commonest abnormality in our series, occurring in nine of 116 cases (7.75\%). The semicircular canals were absent or dysplastic as an isolated finding in three cases (two with the CHARGE association and one VATER RAPADILINO syndrome), in association with a vestibular abnormality in four cases (two were CHARGE cases), and in association with a vestibular and cochlear abnormality in two cases. Semicircular canal and vestibular abnormalities are frequent findings in cases with SNHL, ${ }^{15}$ while deformed or absent semicircular canals is the hallmark of the CHARGE association. ${ }^{18}$ The acronym CHARGE was coined by Pagon et al ${ }^{19}$ to describe the association of colobomata, heart defect, atresia of the choanae, retarded growth/ development, genital hypoplasia, and ear anomalies or deafness.

The CT was abnormal in one of six $(16.6 \%)$ patients with previous documented meningitis. Brookhouser et $a l^{20}$ identified 10 abnormal CT scans in 57 conducted (18\%) on similar cases. CT scan did not identify any abnormalities in either of our two patients with post-traumatic hearing loss.

Profound or progressive hearing loss and craniofacial abnormalities were significant predictors of abnormal CT findings $(\mathrm{p}<0.05)$ and together accounted for 25 of 33 abnormal CT scans (75\%). However, adherence to this joint criterion (presence of profound and/or progressive SNHL and/or craniofacial abnormalities) for CT scanning investigations would have missed one in four cases with abnormal CT scan, or $6.8 \%$ of cases of our total sample.

The CT was instrumental in the aetiological diagnosis in all our cases and helped to clarify 
management issues in most. The CT findings helped substantiate the diagnosis of genetic hearing loss in 21 cases and of a syndromic diagnosis in six, while we are keeping under review the five "congenital" classified cases with abnormal CT scans, as evidence supporting the diagnosis of an autosomal recessive pattern which may emerge in the future. ${ }^{21}$ The above mentioned families have received or are about to receive genetic counselling (apart from a few who declined this offer). Furthermore, additional investigations were initiated in 13 cases, on the basis of the CT results. All three patients with narrow IAM had magnetic resonance imaging (MRI) performed, to identify whether nerve VIII was present and to decide on hearing amplification issues; all 10 cases with DVA were referred for a perchlorate test to exclude Pendred's syndrome. In addition, all patients with DVA were advised to abstain from sports with a high risk of accidents, as their hearing thresholds might deteriorate after trauma. ${ }^{12} 13$

We communicated normal CT findings by letter to patients and discussed them at a regular follow up visit, while abnormal CT findings were personally communicated to parents at an early appointment. We believe that parental stress is reduced and the child's hearing impairment better accepted when we give thorough information on the cause and prognosis of hearing loss for the child and family members. Counselling parents and child is a significant part of the child's management, and CT can provide excellent information for this purpose. CT is the investigation of choice to assess the middle ear. ${ }^{23}$ However, CT may not show the presence of labyrinthitis obliterans reliably in all post-meningitic cases, ${ }^{22}$ or show the presence or absence of nerve VIII, ${ }^{23}$ so, for these purposes, thin section high resolution MRI is indicated. This is particularly the case for cochlear implant candidates, in order to avoid inappropriate cochlear implantation. ${ }^{23}$

CONCLUSION

All children with SNHL must have radiological investigations of the petrous bones/IAMs. The CT scan is more likely to be abnormal in the presence of craniofacial abnormalities, and/or profound or progressive hearing loss. However, not performing CT scan in those with neither profound nor progressive hearing loss, or craniofacial abnormalities may leave up to one of four CT documentable abnormalities un- identified. The decision whether to do a CT or MRI will depend on scanner availability, expertise, and management considerations, but cochlear implant candidates will require both.

We would like to thank Dr Breege MacArdle for her very helpful comments on our findings.

1 European Work Group on Genetics of Hearing Impairment. Hereditary deafness epidemiology and clinical research infoletter. November 1996, No. 2.

2 Zalzal GH, Shott SR, Towbin R, Cotton RT. CT scan in the diagnosis of temporal bone diseases in children. Laryngoscope 1986;96:27-32

3 Shusterman D, Handler SD, Marsh RR, Bilaniuk L, Tom LWC. Usefulness of computed tomographic scan in the evaluation of sensorineural hearing loss in children. Arch Otolaryngol Head Neck Surg 1992;118:501-3.

4 Woolford TJ, Roberts GR, Hartley C, Ramsden RT. Etiology of hearing loss and cochlear computed Etiology of hearing loss and cochlear computed tomography: findings in preimplant asse

5 Auchterlonie IA, White MP. Recurrence of the VATER association within a sibship. Clin Genet 1982;21:122-4.

6 Kant SG, Baraitser M, Milla PJ, Winter RM. Rapadillino syndrome-a non-Finnish case. Clin Dysmorphol 1998;7: $135-8$.

7 Kimberling WJ, Smith RJH. Usher syndrome. In: Martini A, Read A, Stephens D, eds. Genetics and hearing impairment. London: Whurr Publishers, 1996.

8 Martin JAM. Aetiological factors relating to childhood deafness in the European community. Audiology 1982;21: 149-58.

9 Cremers CWRJ, Van Rijn PM, Huygen PLM. The sex-ratio in childhood deafness, an analysis of the male predominance. Int f Pediatr Otorhinolaryngol 1994;30:105-10.

10 Zalzal GH, Tomaski SM, Vezina LG, Bjornsti P, Grundfast KG. Enlarged vestibular aqueduct and sensorineural hearing loss in childhood. Arch Otolaryngol Head Neck Surg 1995;121:23-8.

11 Valvassori GE, Clemis JD. The large vestibular aqueduct syndrome. Laryngoscope 1978;88:723-8

12 Antonelli PJ, Nall AV, Lemmerling MM, Mancuso AA, Kubilis PS. Hearing loss with cochlear modiolar defects and large vestibular aqueducts. Am f Otol 1998;19:306-12.

13 Kou B, Macdonald R. Toronto's Hospital for Sick Children study of traumatic sudden sensorineural hearing loss. $\mathcal{F}$ Otolaryngol 1998;27:64-8.

14 Phelps PD. Mondini and "pseudo Mondini". Clin Otolaryngol 1990;15:99-101.

15 Jackler RK, Luxford WM, House WF. Congenital malformations of the inner ear: a classification based on embryomations of the inner ear: a classificatior

16 Phelps PD, Coffey RA, Trembath RC, et al. Radiological malformation of the ear in Pendred syndrome. Clin Radiol 1998;53:268-73.

17 Chen A, Francis M, Ni L, et al. Phenotypic manifestations of branchio-oto-renal syndrome. Am f Med Genet 1995;58: 365-70

18 Morgan D, Bailey M, Phelps P, Bellman S, Grace A, Wyse R. Ear-nose-throat abnormalities in the CHARGE association. Arch Otolaryngol Head Neck Surg 1993;119:49-54.

19 Pagon RA, Graham JM, Zonana J, Yong S-L. Coloboma, congenital heart disease and choanal atresia with multiple anomalies: CHARGE association. I Pediatr 1981;99:2237 .

20 Brookhouser PE, Auslander MC, Meskan ME. The pattern and stability of postmeningitic hearing loss in children. Laryngoscope 1988;98:940-8.

21 Feinmesser M, Tell L, Levi H. Etiology of childhood deafness with reference to the group of unknown cause. deafness with reference to
Audiology 1986;25:65-9.

22 Phelps PD, Proops DW. Imaging for cochlear implants. $f$ Laryngol Otol 1999;113:21-3.

23 Phelps PD. Imaging for cochlear implants: the importance of the latest techniques for preoperative assessment. $f$ Audiol Med 1998;7:145-54. 